\title{
Motor Adaptation during Slip-Perturbed Gait in Older Adults
}

\author{
Misono SAKAI ${ }^{1)}$, YoshitaKa SHIBA ${ }^{2)}$, HARUHIKO SATO ${ }^{2)}$, NAONOBU TAKAHIRA ${ }^{1,2)}$ \\ 1) Graduate School of Medical Science, Kitasato University: 1-15-1 Kitasato, Sagamihara, \\ Kanagawa 228-8555, Japan. TEL +81 42-778-9568, FAX +81 42-778-9568, \\ E-mail:dm04023w@st.kitasato-u.ac.jp \\ ${ }^{2)}$ Department of Allied Health Sciences, Kitasato University
}

J. Phys. Ther. Sci.

20: 109-115, 2008

\begin{abstract}
Purpose] The purpose of this study was to investigate adaptations to slip perturbations using a customized separated-belt treadmill in older adults. [Subjects] Forty-five healthy older subjects participated in this study. [Methods] Participants were requested to continue to walk against 20 slip perturbations during a 5-minute period. The magnitudes of the reactive responses were evaluated with the use of a tri-axial accelerometer attached to the subject's pelvis. Also, the latency to the onset of EMG activity and the magnitude of EMG activity were measured using surface electrodes placed on both sides of the erector spinae, rectus abdominis, biceps femoris, vastus medialis (VM), gastrocnemius medialis (GM), and tibialis anterior muscles. [Results] The magnitude of the reactive response in the second half of the perturbed walk was significantly more decreased than during the first half $(p<0.01)$. Decreases in the magnitude of EMG were also observed from VM and GM muscles ( $p<0.01, p<0.05$, respectively), but the latency of each of the muscles was unchanged. [Conclusion] In conclusion, adaptations that may have contributed to the prevention of falls occurred in older adults. These adaptations might have been accomplished by a change in magnitude of muscle activity rather than by a change in timing.
\end{abstract}

Key words: Treadmill-walking, Balance, Muscle activity

(This article was submitted Oct. 11, 2007, and was accepted Nov. 30, 2007)

\section{INTRODUCTION}

Falls are seen more often in older people than among younger people, and they have been attracting concern among the growing elderly population. In 2005, over 1800 elderly people in Japan died due to fall-related injuries at home ${ }^{1)}$. Fractures caused by falls were the third most common reason for people to need nursing in $\mathrm{Japan}^{2)}$. Therefore, preventing falls is one of the key factors for maintaining the quality of life in older adults.

Older adults tend to show muscle weakness and diminished sensory functions to one degree or another ${ }^{3)}$, but the relationship between these factors and the occurrence of falls is still unknown. Muscle strengthening exercises for the elderly have been used in fall prevention programs, but no clear association was found, especially in the healthy elderly, between the occurrence of falls and muscle strength. Nowalk et al. reported that an exercise program that included muscle strengthening was not effective for improving fall rates ${ }^{4)}$. In a retrospective study, older fallers showed a poorer two-point discrimination sense than that of older non-fallers ${ }^{5)}$, but proprioceptive training ${ }^{6,7)}$ has not been widely accepted in fall prevention programs because of limited evidence that it is effective in lowering the incidence of falls.

Repeated perturbation exposure may reduce the incidence of falls ${ }^{8)}$. Healthy older adults appeared fully capable of learning to recover better from, or 
adjusting to, repeated exposures to perturbations during a sit-to-stand task ${ }^{9-11)}$. Smith et al. ${ }^{12)}$ examined whether a difference exists between muscle responses of older fallers and those of nonfallers during repeated dorsi-flexion perturbations to standing. Their results showed that an adaptive response, characterized by attenuation in the magnitude of muscle responses, was observed only in the non-fallers. Moreover, Obuchi et al. showed that long-term improvements in balance among older adults was achieved by treadmill walking with repeated slip perturbations ${ }^{8}$.

Because most falls occur when a person is walking, adaptive responses to slip perturbations during walking may be effective for preventing falls. The purpose of this study was to confirm whether older adults would develop better recoveries from a slip during walking by responding to repeated perturbations. We also examined the effects on the latency and magnitude of muscle responses in the trunk, thigh, and leg muscles.

\section{METHODS}

\section{Subjects}

Forty-five community-dwelling elderly subjects (71.4 \pm 3.6 years, $56.5 \pm 10.5 \mathrm{~kg}, 154.6 \pm 7.9 \mathrm{~cm}, 19$ males and 26 females) participated in this study. Subjects had no history of neurological or musculoskeletal disorders, and only one woman had fallen in the past year. We gave information about this research to all subjects and obtained their written agreements to participation before starting the experiment. This experiment was approved by the Research Ethics Committee of The Society of Physical Therapy Science.

\section{Procedures}

We used a separated-belt treadmill ${ }^{13)}$ (PW21; Hitachi, Japan), and perturbations were produced by rapidly decelerating the right side of the walking belt for $500 \mathrm{msec}$ while subjects were walking. After $500 \mathrm{msec}$ the speed of the walking belt was returned to its initial level. By decelerating the walking belt, the base of support (BOS) of the stance phase leg became located in front (unlike before the deceleration), and the body swayed backward. All subjects walked on the treadmill for 5 minutes at $2 \mathrm{~km} / \mathrm{h}$ looking ahead without holding onto a rail. In addition, bipolar surface electrodes of electromyogram (EMG) (Neuropack8; MEB-4208,
NIHON KOHDEN, Japan) and an accelerometer (HITACHI, Japan) were attached. The perturbation was a $50 \%$ deceleration from the initial speed ( 2 $\mathrm{km} / \mathrm{h}$ ) and was given 20 times repeatedly during heel contact as it occurred in the 5-minute walk. The perturbations were delivered to the treadmill from a PC before heel contact without a cue providing notice, causing the walking belt to decelerate when the heel contacted the belt. Two assistants stood on both sides of the subjects to prevent falls and injuries. The heel contacts were detected by a force plate under the treadmill, and all data were synchronized by an A/D converter and sampled at $1000 \mathrm{~Hz}$ (Fig. 1).

\section{Data analyses}

The treadmill was supported by only 4 rods, each put on one force plate. The sum $\mathrm{Fz}$ of plates 1 and 4 was the vertical force on the right side of the treadmill; the sum $\mathrm{Fz}$ of plates 2 and 3 was the vertical force on the left side (Fig. 1). The raw data of the force plates were smoothed based on the filter of Bryant ${ }^{14)}$. We detected heel contacts by reference to $\mathrm{Fz}$ of plate 1 because perturbations were given to the right side, and subjects placed their heel on plate 1 . Heel contact was defined as when the value of $\mathrm{Fz}$ of plate 1 shifted from a decrease to an increase.

EMG signals were recorded from the erector spinae (ES) at the level of Th10, rectus abdominis (ABD) at the level of the umbilicus, biceps femoris (BF), vastus medialis (VM), gastrocnemius medialis (GM), and tibialis anterior (TA) muscles of both legs. The distance between the bipolar surface electrodes was $3 \mathrm{~cm}$ on each muscle. These electrodes were placed above the midpoint of the muscle belly, parallel to the direction of the muscle fibers. The signals were analog-filtered at 10-1000 Hz. Full-wave EMG signals on the perturbed side were analyzed for latency and integral EMG (IEMG). The latency reflected the time before the muscle reaction, and the IEMG reflected the EMG amplitude. All latencies in the muscle activations were determined as the duration from $50 \mathrm{msec}$ after the perturbation to a threshold value of muscle EMG. Each muscle's threshold value was defined as the mean +3 S.D. of the muscle activation immediately before the perturbation on a one-gait cycle. All IEMG data were analyzed on a perturbed one-gait cycle (Perturbed IEMG), a normal one-gait cycle immediately before perturbation (Normal 


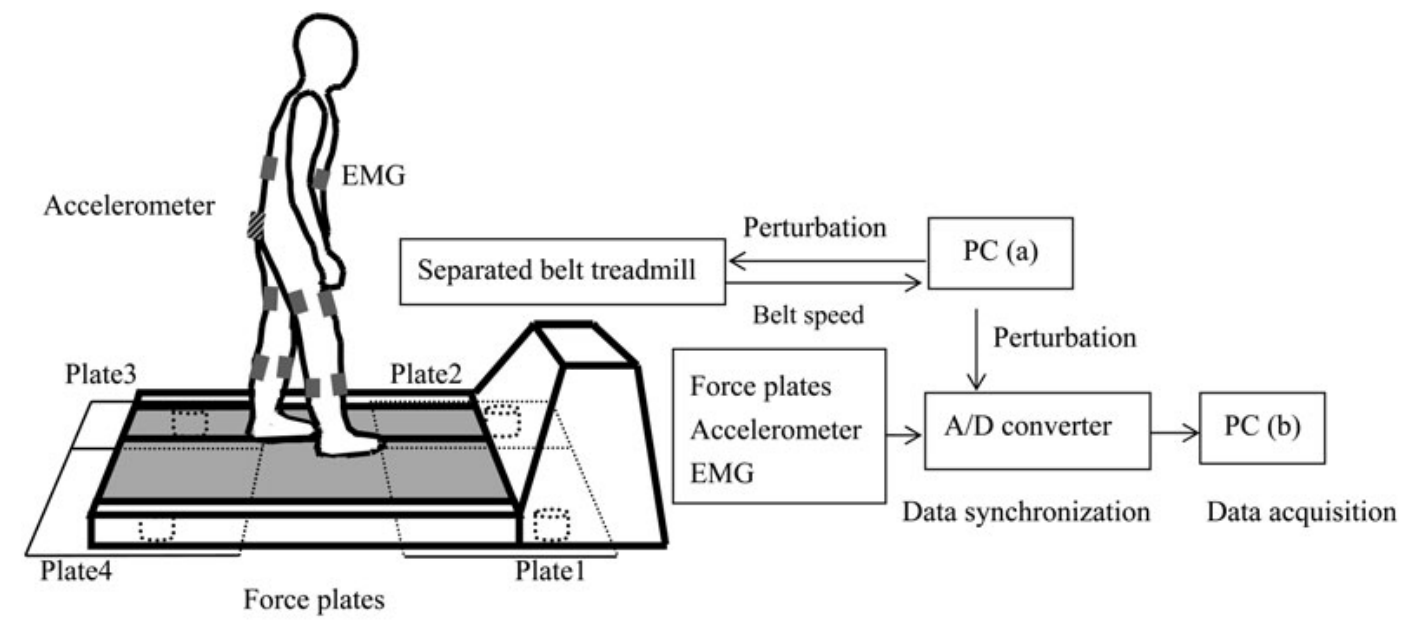

Fig. 1. Experimental system. The perturbations are delivered from PC (a) to the treadmill. The treadmill is supported by only 4 rods, one on each force plate. Heel contacts were detected from force plate 1 under the treadmill. Force plate, accelerometer, EMG, and perturbation signals are synchronized by an A/D converter, and then the data are delivered to PC (b).

IEMG), and a Perturbed IEMG divided by a Normal IEMG (Ratio IEMG) (Fig. 2). Therefore, if the value of Ratio IEMG exceeded 1, then the amplitude of the muscle activation on a perturbed one-gait cycle was larger than that of the muscle activation on a normal one-gait cycle.

The accelerometer was set in the center of the sacrum to measure the acceleration (ACC) of the pelvis and reflected the degree of body sway. The ACC was analyzed for the maximum value of the forward and backward movements of the pelvis after the perturbation (Fig. 2). A larger value of the ACC meant a larger body sway.

Data from 20 trials, obtained for each subject, were divided equally into 10 trials in the first half and 10 trials in the second half. The average for each subject for the ACC, stride time, IEMG, and latency values were compared between the first and second halves.

Statistical analyses were performed with the SPSS $11.0 \mathrm{~J}$ for Windows software. The average of ACC, stride time, IEMG, and latency values in the first and second halves were assessed by the Wilcoxon matched pairs signed rank test; statistical significance was considered as $\mathrm{p}<0.05$.

\section{RESULTS}

All elderly subjects could walk on the treadmill and responded to 20 trial perturbations with no holding onto the rail. Subjects' performances improved as the trials advanced. In fact, the maximum value of the ACC of both forward and backward directions decreased as the trials advanced. In addition, the maximum value in the second half was significantly smaller than during the first half for both the forward and backward movements $(\mathrm{p}<0.01)$ (Table 1) (Forward: first half $1.26 \pm 0.50 \mathrm{G}$, second half $1.17 \pm 0.43 \mathrm{G}$; Backward: first half $2.89 \pm 1.33 \mathrm{G}$, second half 2.68 $\pm 1.25 \mathrm{G})$. These results seem to suggest that the sway of the body became smaller as the trials advanced. The stride time of the normal one-gait cycle was not significantly changed, but the perturbed one-gait cycle in the second half significantly increased from the value in the first half (Normal one-gait cycle: $+1.3 \mathrm{msec}$, not significant; Perturbed one-gait cycle: $+37.6 \mathrm{msec}$, $\mathrm{p}<0.01$ ) (Table 2).

Table 1 shows the EMG data. For the Ratio IEMG, TA had the greatest reaction, followed by VM (TA: first half $3.13 \pm 1.58$, second half $3.09 \pm$ 1.35; VM: first half $1.73 \pm 0.46$, second half $1.65 \pm$ 0.45 ). GM had the smallest reaction, and only GM showed a value below 1 (first half $0.98 \pm 0.28$, second half $0.93 \pm 0.25$ ) (Table 1). This means that for GM, the Perturbed IEMG was smaller than the Normal IEMG. The Ratio IEMGs of ABD, VM, and GM in the second half were significantly decreased from the first half. Especially, the Perturbed IEMGs of VM and GM were significantly decreased in the second half, but the 


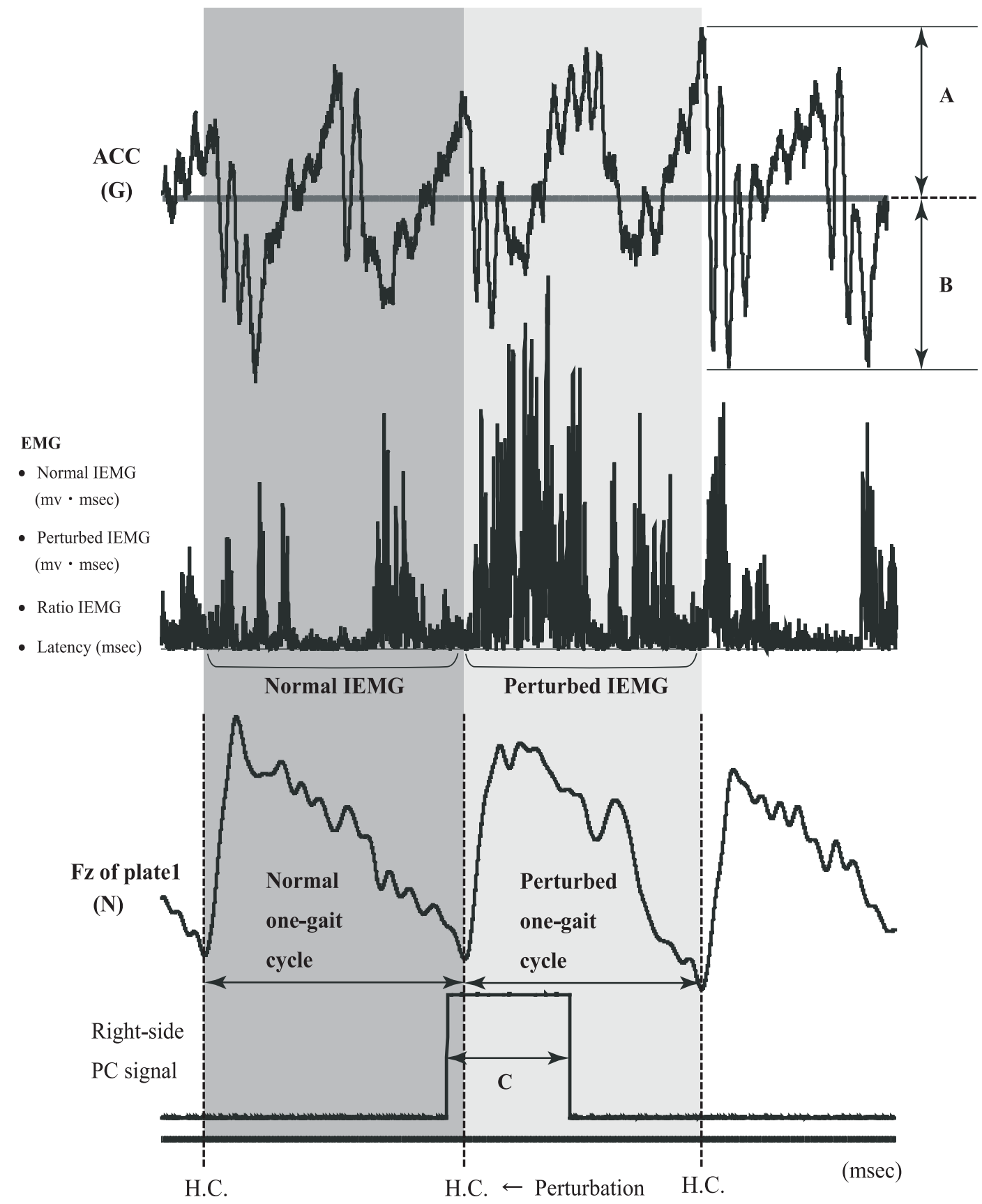

Fig. 2. Data analysis for each trial. A: Maximum value of posterior motion of the accelerometer on the pelvis after perturbation. B: Maximum value of anterior motion of the accelerometer on the pelvis after perturbation. C: Deceleration of right-side belt speed. ACC: acceleration. H.C.: Heel contact on the right side. The heel contact was defined as when the value of Fz for plate 1 changed from a decreasing to an increasing value. Perturbed IEMG: Integral EMG of a perturbed one-gait cycle. Normal IEMG: Integral EMG of a normal one-gait cycle immediately before perturbation. Ratio IEMG: Perturbed IEMG divided by Normal IEMG.

Normal IEMGs for these muscles were not (VM: Normal IEMG $-1.45 \mathrm{mV} \cdot \mathrm{msec}$, not significant; Perturbed IEMG $-5.8 \mathrm{mV} \cdot \mathrm{msec}, \mathrm{p}<0.01)(\mathrm{GM}$ : Normal IEMG $+0.48 \mathrm{mV} \cdot \mathrm{msec}$, not significant;
Perturbed IEMG -4.46 mV·msec, $\mathrm{p}<0.05$ ) (Table1). The Ratio IEMG of TA was not significantly changed, but the Normal IEMG and Perturbed IEMG in the second half were significantly 
Table 1. Average of ACC, IEMG, and latency

\begin{tabular}{|c|c|c|c|c|c|c|}
\hline & & & & $\begin{array}{c}\text { Ten trials } \\
\text { in the first half }\end{array}$ & $\begin{array}{l}\text { Ten trials } \\
\text { in the second half }\end{array}$ & significant \\
\hline \multirow[t]{2}{*}{$\mathrm{ACC}$} & \multirow{2}{*}{\multicolumn{2}{|c|}{ Maximum value(G) }} & forward & $1.26 \pm 0.50$ & $1.17 \pm 0.43$ & $* *$ \\
\hline & & & backward & $2.89 \pm 1.33$ & $2.68 \pm 1.25$ & $* *$ \\
\hline \multirow[t]{24}{*}{ EMG } & IEMG & Ratio IEMG: & ES & $1.37 \pm 0.45$ & $1.32 \pm 0.41$ & n.s. \\
\hline & \multirow[t]{17}{*}{ (1gait cycle IEMG) } & \multirow[t]{5}{*}{ (Perturbed/Normal) } & $\mathrm{ABD}$ & $1.31 \pm 0.33$ & $1.25 \pm 0.30$ & $*$ \\
\hline & & & $\mathrm{BF}$ & $1.40 \pm 0.50$ & $1.35 \pm 0.45$ & n.s. \\
\hline & & & VM & $1.73 \pm 0.46$ & $1.65 \pm 0.45$ & $*$ \\
\hline & & & GM & $0.98 \pm 0.28$ & $0.93 \pm 0.25$ & $*$ \\
\hline & & & TA & $3.13 \pm 1.58$ & $3.09 \pm 1.35$ & n.s. \\
\hline & & \multirow{6}{*}{$\begin{array}{l}\text { Normal IEMG } \\
(\mathrm{mV} \cdot \mathrm{msec})\end{array}$} & ES & $51.71 \pm 40.46$ & $52.24 \pm 39.38$ & n.s. \\
\hline & & & $\mathrm{ABD}$ & $19.48 \pm 12.07$ & $20.58 \pm 12.45$ & n.s. \\
\hline & & & $\mathrm{BF}$ & $63.91 \pm 49.74$ & $62.82 \pm 46.23$ & n.s. \\
\hline & & & VM & $45.40 \pm 35.81$ & $43.95 \pm 34.52$ & n.s. \\
\hline & & & GM & $101.40 \pm 71.22$ & $101.88 \pm 70.94$ & n.s. \\
\hline & & & TA & $74.54 \pm 52.13$ & $64.37 \pm 39.79$ & $*$ \\
\hline & & \multirow{6}{*}{$\begin{array}{l}\text { Perturbed IEMG } \\
(\mathrm{mV} \cdot \mathrm{msec})\end{array}$} & ES & $66.36 \pm 44.66$ & $65.28 \pm 42.63$ & n.s. \\
\hline & & & $\mathrm{ABD}$ & $24.13 \pm 15.14$ & $24.59 \pm 15.55$ & n.s. \\
\hline & & & $\mathrm{BF}$ & $79.57 \pm 57.72$ & $76.77 \pm 52.47$ & n.s. \\
\hline & & & VM & $71.53 \pm 46.55$ & $65.73 \pm 43.25$ & $* *$ \\
\hline & & & GM & $93.70 \pm 67.24$ & $89.24 \pm 61.62$ & $*$ \\
\hline & & & TA & $209.89 \pm 149.96$ & $181.92 \pm 119.28$ & $*$ \\
\hline & \multirow[t]{6}{*}{ Latency (msec) } & & ES & $244.2 \pm 108.9$ & $274.3 \pm 117.9$ & n.s. \\
\hline & & & $\mathrm{ABD}$ & $304.1 \pm 112.8$ & $303.9 \pm 101.8$ & n.s. \\
\hline & & & $\mathrm{BF}$ & $167.5 \pm 83.7$ & $191.4 \pm 114.8$ & n.s. \\
\hline & & & VM & $87.3 \pm 27.9$ & $88.0 \pm 29.4$ & n.s. \\
\hline & & & GM & $336.7 \pm 148.1$ & $347.8 \pm 143.1$ & n.s. \\
\hline & & & TA & $139.1 \pm 45.0$ & $136.5 \pm 33.5$ & n.s. \\
\hline
\end{tabular}

Values are means \pm S.D. for 45 older subjects.

ACC: acceleration; IEMG: Integral EMG; ES: erector spinae; ABD: rectus abdominis; BF: biceps femoris; VM: vastus medialis; GM: gastrocnemius medialis; TA: tibialis anterior; *: $p<0.05$, **: $p<0.01$, n.s.: not significant.

Table 2. Average of the stride time in the first half and the second half of the trials

\begin{tabular}{lccc}
\hline & Ten trials in the first half & Ten trials in the second half & significant \\
\hline Normal one-gait cycle $(\mathrm{msec})$ & $1069.3 \pm 139.4$ & $1070.6 \pm 140.5$ & n.s. \\
Perturbed one-gait cycle $(\mathrm{msec})$ & $1075.9 \pm 177.7$ & $1113.5 \pm 185.1$ & $* *$ \\
\hline
\end{tabular}

Values are means \pm S.D. for 45 older subjects. $* *$ : $p<0.01$, n.s.: not significant.

decreased from their values in the first half (TA: Normal IEMG $-10.17 \mathrm{mV} \cdot \mathrm{msec}, \mathrm{p}<0.05$; Perturbed IEMG $-27.97 \mathrm{mV} \cdot \mathrm{msec}, \mathrm{p}<0.05)($ Table 1$)$. Regarding the latency, VM reacted the fastest, followed by TA (VM: first half $87.3 \pm 27.9 \mathrm{msec}$, second half $88.0 \pm 29.4 \mathrm{msec}$; TA: first half $139.1 \pm$ $45.0 \mathrm{msec}$, second half $136.5 \pm 33.5 \mathrm{msec}$ ). There were no significant changes between the first and second halves regarding the latencies for all the muscles. This suggests that the amplitude of muscle activations rather than the latency affected the motor adaptation for postural control.

\section{DISCUSSION}

In this study, we demonstrated motor adaptations for postural control for repeated perturbations during treadmill walking in elderly subjects. The maximum of the acceleration for both forward and backward movements in the second half of the trials became significantly smaller than those in the first half. This means that the body sway of the elderly subjects became smaller as the trials advanced. The decrease in body sway indicates that elderly subjects can adapt by improving their postural 
control in response to repeated perturbations during walking. For perturbations in this research, the Ratio IEMG of TA was the biggest, and the latency of TA was shorter than those of the other muscles. This was evidenced in subjects' postural control for slip perturbations during standing and walking. In "ankle strategy" during standing, activities began with TA and were followed by the quadriceps and rectus abdominis ${ }^{15)}$. This pattern was also shown during walking ${ }^{16)}$. For these reasons, we think that effective responses by TA are required for postural control in slip perturbations during walking.

We were able to determine that the causes of improvement in postural control were a decreased Integral EMG of some muscles. On the other hand, the latency had no effect on the immediate adaptation for postural control in this research. Perturbed IEMGs of VM and GM were significantly decreased, but the Normal IEMGs for these muscles were not. We think that VM and GM reacted more than the required degree during the first half. Their hyper-reaction made it possible to hinder the required muscle response and resulted in stiffer joint movements and hindered quick reactions, so body sway in the first half of the trials was bigger than that in the second half. We noted especially that the excessive reaction of GM appeared to induce too much co-contraction between TA and GM and hindered TA response in the first half of the trials. However, because the GM magnitude was attenuated in the second half, the TA responded more effectively in the second half producing an elastic ankle movement. In other words, reciprocal inhibition between TA and GM became more effective in the second half of the trials. We think that the older subjects in this research showed too much co-activation in the first half, but their reciprocal inhibition gradually became effective and the body sways smaller in the second half.

This trend of co-activation in postural control with perturbations among older subjects during walking has already been reported ${ }^{16)}$. In addition, an attenuation in the muscle response magnitude from perturbations as postural control improved has been shown in walking of young subjects ${ }^{17)}$, and during standing among the non-faller elderly ${ }^{12}$. Therefore, we think that people, especially older adults, have a tendency to have exaggerated muscle response magnitude and co-activation from unknown perturbations, but can adapt to repeated perturbations by a method of attenuating muscle activity and co-contractions that would otherwise be excessive.

We did not see an improvement in muscle latency in this study. However, subjects participating in long-term treadmill walking that included training with perturbations ${ }^{8)}$ and sensorimotor training ${ }^{6}$ ) showed decreased muscle latency. Therefore, we suspect that long-term, as opposed to short-term, training can improve muscle latency. In this research, we investigated only muscle responses to clarify ways of adaptation. However, there is the possibility that elderly subjects can adapt by feedforward control to compensate for repeated perturbations - for example, changing the center of mass (COM) location and the movement of upper limbs, as has been shown in several reports ${ }^{9-11,18-20)}$. In addition, we think that change of head movement occurred. Head movements and corresponding vestibular input influence postural response ${ }^{21)}$. In the case of repeated perturbation to seated subjects' trunks, the subjects attenuated their neck muscle activity and modulated their head movement ${ }^{22}$. Changes in the muscle responses seen in this study might have been a result of these other strategies. Therefore, we need to make other kinetic analyses and study the influence of exposure to perturbations over a long-term period.

In conclusion, older adults could adapt to repeated perturbations during walking. Adjusting the force control, particularly the coordination between agonist and antagonistic muscles, is an effective strategy for adaptation that contributes to preventing falls. This strategy may become a key to training for fall prevention.

\section{ACKNOWLEDGEMENTS}

The authors thank Dr. Shuichi Obuchi who created the treadmill used in this study and supported this research. In addition, we are very grateful to all the subjects who participated in the experiment and to all the staff members who helped with data collection.

\section{REFERENCES}

1) Ministry of Health, Labour and Welfare, Japan: Vital Statistics of Japan 2005 volume 3. Tokyo: Health and Welfare Statistics Association, 2007, p522 (in Japanese). 
2) Ministry of Health, Labour and Welfare, Japan: Kokumin Seikatsu Kiso Chousa 2004 volume 1. Tokyo: Health and Welfare Statistics Association, 2006, p201 (in Japanese).

3) Tang PF, Woollacott MH: Balance control in older adults. In: Clinical Disorders of balance, Posture and gait: second edition. Bronstein AM, Brandt T, Woollacott MH, et al. (eds), London: Arnold, 2004, pp385-403.

4) Nowalk MP, Prendergast JM, Bayles CM, et al.: A randomized trial of exercise programs among older individuals living in two long-term care facilities: The FallsFREE program. J Am Geriatr Soc, 2001, 49: 859865.

5) Melzer I, Benjuya N, Kaplanski J: Postural stability in the elderly: A comparison between fallers and nonfallers. Age Ageing, 2004, 33: 602-607.

6) Granacher U, Gollhofer A, Strass D: Training induced adaptations in characteristics of postural reflexes in elderly men. Gait Posture, 2006, 24: 459-466.

7) Sinaki M, Brey RH, Hughes CA, et al.: Significant reduction in risk of falls and back pain in osteoporotickyphotic women through a spinal proprioceptive extension exercise dynamic (SPEED) program. Mayo Clin Proc, 2005, 80: 849-855.

8) Obuchi S, Kojima M, Shiba Y, et al.: A randomized controlled trial of a treadmill training with the perturbation to improve the balance performance in the community dwelling elderly subjects. Nippon Ronen Igakkai Zasshi, 2004, 41: 321-327 (in Japanese with English Abstract).

9) Pai YC, Wening JD, Runtz EF, et al.: Role of feedforward control of movement stability in reducing slip-related balance loss and falls among older adults. J Neurophysiol, 2003, 90: 755-762.

10) Pavol MJ, Runtz EF, Edwards BJ, et al.: Age influences the outcome of a slipping perturbation during initial but not repeated exposures. J Gerontol A Biol Sci Med Sci, 2002, 57: M496-503.

11) Pavol MJ, Runtz EF, Pai YC: Young and older adults exhibit proactive and reactive adaptations to repeated slip exposure. J Gerontol A Biol Sci Med Sci, 2004,
59: 494-502.

12) Smith BN, Segal RL, Wolf SL: Long latency ankle responses to dynamic perturbation in older fallers and non-fallers. J Am Geriatr Soc, 1996, 44: 1447-1454.

13) Hase $K$, Obuchi $S$, Horie $T$ : Rehabilitation system to prevent falls during walking in older adults (Planning of design concept and primary experiments). Transactions of the Japan Society of Mechanical Engineers, Series C, 2002, 68: 1245-1250 (in Japanese with English Abstract).

14) Bryant JT, Wevers HW, Lowe PJ: Methods of data smoothing for instantaneous centre of rotation measurements. Med Biol Eng Comput, 1984, 22: 597602.

15) Horak FB, Nashner LM: Central programming of postural movements: Adaptation to altered supportsurface configurations. J Neurophysiol, 1986, 55: 1369-1381.

16) Tang PF, Woollacott MH: Inefficient postural responses to unexpected slips during walking in older adults. J Gerontol A Biol Sci Med Sci, 1998, 53: M471-480.

17) Marigold DS, Patla AE: Strategies for dynamic stability during locomotion on a slippery surface: Effects of prior experience and knowledge. J Neurophysiol, 2002, 88: 339-353.

18) Bhatt T, Pai YC: Long-term retention of gait stability improvements. J Neurophysiol, 2005, 94: 1971-1979.

19) Bhatt T, Wening JD, Pai YC: Adaptive control of gait stability in reducing slip-related backward loss of balance. Exp Brain Res, 2006, 170: 61-73.

20) Pavol MJ, Pai YC: Feedforward adaptations are used to compensate for a potential loss of balance. Exp Brain Res, 2002, 145: 528-538.

21) Horak FB, Shupert CL, Dietz V, et al.: Vestibular and somatosensory contributions to response to head and body displacements in stance. Exp Brain Res, 1994, 100: 93-106.

22) Blouin JS, Descarreaux M, Belanger-Gravel A, et al.: Attenuation of human neck muscle activity following repeated imposed trunk-forward linear acceleration. Exp Brain Res, 2003, 150: 458-464. 